\title{
Noncommutative Orlicz spaces associated to a state
}

\author{
by
}

\section{Maryam H. A. Al-Rashed and BogusŁaw Zegarliński (London)}

\begin{abstract}
We introduce and study the noncommutative Orlicz spaces associated to a normal faithful state on a semifinite von Neumann algebra.
\end{abstract}

1. Introduction. The main objective of this paper is to establish the basis of the theory of noncommutative Orlicz spaces associated to a faithful normal state on a semifinite von Neumann algebra. The family of spaces we introduce generalises the noncommutative $L_{p}$ spaces, and is furnished with Orlicz and Luxemburg norms, which - as we prove - are equivalent. Moreover we show that the suitable Young and Hölder inequalities are true. We note that in the case of Orlicz spaces associated to a trace such a theory was presented long time ago in [12] (see also [13], [14]). Its special case, the noncommutative $L_{p}$ spaces associated to a nontrivial state, was studied extensively even earlier (see [2], [5], [7]-[9], [11], [15], [18], [19]-[22]). Strictly speaking, given the Orlicz spaces for traces (as in [12]), by using the noncommutative integration theory an abstract and complicated construction of Orlicz spaces is possible even when working with factor III type von Neumann algebras. What we demonstrate is that, with a bit of extra technical assumptions at the start, one can introduce the Orlicz spaces in a very explicit way, which in the special case of monomial type Young functions reproduces the original noncommutative $L_{p}$ spaces. We believe that such explicit representation is useful not only to study interesting properties of noncommutative Orlicz spaces, but should also be of great potential interest for various applications.

The paper is organised as follows. In the first section we introduce notation and recall some known facts from the theory of classical Orlicz spaces with respect to a given measure [16], as well as the noncommutative analog

2000 Mathematics Subject Classification: 46B28, 46L51, 46L52.

Key words and phrases: Orlicz space, von Neumann algebra, faithful normal trace.

M. H. A. Al-Rashed on leave from Department of Mathematics, Basic College of Education, Kuwait. 
involving the trace in place of integration [12]. In the next section we introduce a definition of the Orlicz space associated to a faithful normal state on a semifinite von Neumann algebra furnished with the analog of Orlicz and Luxemburg norms. In that section we also prove the Young and Hölder inequalities, and equivalence of various norms. We finish with a brief summary and outlook.

2. Preliminaries. A continuous, convex, nonnegative, strictly increasing function $\Phi$ of a real variable is called a Young function iff for any $x \in \mathbb{R}$,

$$
\Phi(x)=\int_{0}^{|x|} \varphi(t) d t
$$

with a function $\varphi:[0, \infty) \rightarrow \mathbb{R}^{+}$which vanishes only at zero, is right continuous, nondecreasing and diverges to infinity as $t \rightarrow \infty$. By this definition, $\Phi$ is unbounded and vanishes only at zero. For every Young function $\Phi$ there exists a function $\Psi$, called the complementary Young function, given as follows:

$$
\Psi(x)=\int_{0}^{|x|} \psi(s) d s, \quad x \geq 0,
$$

where $\psi$ is the right inverse of $\varphi$. A pair of complementary Young functions $(\Phi, \Psi)$ satisfies the following Young inequality:

$$
t s \leq \Phi(t)+\Psi(s) \quad \text { for all } t, s \in[0, \infty),
$$

with equality if and only if $t=\psi(s)$ or $s=\varphi(t)$ (see [10] or [16]). We remark that the class of Young functions generalises the well known family of monomial type functions $x \mapsto(1 / p) x^{p}, p \geq 1$.

For later purposes we note that the inverse functions $\Phi^{-1}$ and $\Psi^{-1}$ satisfy

$$
r<\Phi^{-1}(r) \Psi^{-1}(r) \leq 2 r
$$

for any nonnegative $r$ (see [16]).

Let $\mathcal{A}$ be a semifinite von Neumann algebra on a Hilbert space $\mathcal{H}$ with a faithful normal semifinite trace $\operatorname{Tr}$. We denote by $\mathcal{M}=\mathcal{M}(\mathcal{A})$ the topological *-algebra (equipped with the measure topology) of trace-measurable operators [15], [22]. [Recall that a closed densely defined operator $f$ affiliated with $\mathcal{A}$ is called trace-measurable iff, for each $\varepsilon \in \mathbb{R}^{+}$, there exists a projection $p \in \mathcal{A}$ such that

$$
p \mathcal{H} \subseteq \mathcal{D}(f) \text { and } \operatorname{Tr}(1-p) \leq \varepsilon,
$$

where $\mathcal{D}$ is the domain of $f$. The measure topology on $\mathcal{M}(\mathcal{A})$ is by definition the linear topology in which the sets

$$
\begin{aligned}
& V(\varepsilon, \delta)=\{a \in \mathcal{M}(\mathcal{A}): \exists \text { projection } q \in \mathcal{A},\|a q\|<\varepsilon \text { and } \operatorname{Tr}(1-q)<\delta\} \\
& \text { with } \varepsilon, \delta \in \mathbb{R}^{+} \text {, form a base of neighbourhoods of zero.] }
\end{aligned}
$$


A closed densely defined operator $f$ affiliated with $\mathcal{A}$ has a polar decomposition $f=u|f|$ with a partial isometry $u$ and modulus $|f|$ of $f$ admitting the spectral decomposition $|f|=\int_{0}^{\infty} \lambda d e_{\lambda}$. The rearrangement $\widetilde{f}$ of $f$ is by definition the function given by

$$
\widetilde{f}(t):=\inf \left\{\lambda>0: \operatorname{Tr}\left(e_{(\lambda, \infty)}\right) \leq t\right\}
$$

where $e_{(\lambda, \infty)}$ denotes the corresponding spectral projector of $|f|$. Following [6] and [22] we list the following properties of the rearrangement mapping.

Lemma 2.1. Let $A \in \mathcal{M}(\mathcal{A})$. Then:

(1) $\widetilde{A}=\widetilde{A^{*}}=|A|^{\sim}$.

(2) If $X$ is a bounded measurable operator, then $\widetilde{A X} \leq\|X\| \widetilde{A}$.

(3) If $B \in \mathcal{M}(\mathcal{A})$ and $0 \leq A \leq B$, then $\widetilde{A} \leq \widetilde{B}$.

(4) For $0 \leq A \in \mathcal{M}(\mathcal{A})$ and any continuous increasing function $\phi$ : $[0, \infty) \rightarrow[0, \infty)$ one has $\phi(\widetilde{A})=\widetilde{\phi(A)}$ and

$$
\operatorname{Tr}(\phi(A))=\int_{0}^{\infty} \phi(\widetilde{A}(t)) d t .
$$

For later use we also recall the following properties of operator absolute value (cf [1], [4] and [3], [12]).

\section{LEMMA 2.2 .}

(1) (Operator triangle inequality) For any $A, B \in \mathcal{M}(\mathcal{A})$ there exist partial isometries $u, v \in \mathcal{A}$ with $u u^{*}=v v^{*}=1$ such that

$$
|A+B| \leq u^{*}|A| u+v^{*}|B| v .
$$

(2) (Jensen inequality for traces) If $\Phi$ is a Young function and $z \in$ $\mathcal{M}(\mathcal{A}),\|z\| \leq 1$, then for any nonnegative measurable $f$ one has

$$
\operatorname{Tr}\left(\Phi\left(z^{*} f z\right)\right) \leq \operatorname{Tr}\left(z^{*} \Phi(f) z\right) .
$$

(3) (Young inequality for traces) For any $A, B \in \mathcal{M}(\mathcal{A})$ and a pair of complementary Young functions $\Phi$ and $\Psi$ one has

$$
\operatorname{Tr}(|A B|) \leq \operatorname{Tr}(\Phi(|A|))+\operatorname{Tr}(\Psi(|B|)) .
$$

\section{Noncommutative Orlicz spaces associated to a faithful normal} state. Let $\operatorname{Tr}$ be a faithful trace on a semifinite von Neumann algebra $\mathcal{A}$ and let $\omega$ be a state given by

$$
\omega(f)=\operatorname{Tr}(\varrho f)
$$

with a measurable density operator $\varrho>0$ (normalisation is nowhere important). For any Young function $\Phi$ and a given $s \in[0,1]$ we define the Orlicz functional

$$
\mathcal{M}(\mathcal{A}) \ni f \mapsto O_{\Phi, s}(f):=\operatorname{Tr}\left(\Phi\left(\left|F_{\Phi, s}\right|\right)\right)
$$


with

$$
F_{\Phi, s}:=\left(\Phi^{-1}(\varrho)\right)^{s} f\left(\Phi^{-1}(\varrho)\right)^{1-s}
$$

Define

$$
K_{\Phi, s}=\left\{f \in \mathcal{M}(\mathcal{A}): O_{\Phi, s}(f) \leq 1\right\}, \quad L_{\Phi, s}(\mathcal{A}, \omega):=\bigcup_{n=1}^{\infty} n K_{\Phi, s} .
$$

One can see that $h \in L_{\Phi, s}(\mathcal{A}, \omega)$ iff there exists $\beta \in(0,1]$ such that $\beta h \in$ $K_{\Phi, s}$. We have the following two properties.

\section{Proposition 3.1.}

(i) $K_{\Phi, s}$ is absolutely convex.

(ii) $L_{\Phi, s}(\mathcal{A}, \omega)$ is a linear space.

Proof. (i) We need to show that for $\alpha \in[0,1]$ and all $f, g \in K_{\Phi, s}$, we have $\alpha f+(1-\alpha) g \in K_{\Phi, s}$. To this end we note that by Lemma 2.2, there are partial isometries $u$ and $v$ with $u u^{*}=v v^{*}=1$ such that

$$
|\alpha F+(1-\alpha) G| \leq \alpha u^{*}|F| u+(1-\alpha) v^{*}|G| v,
$$

where

$$
\begin{aligned}
F & \equiv F_{\Phi, s} \equiv\left(\Phi^{-1}(\varrho)\right)^{s} f\left(\Phi^{-1}(\varrho)\right)^{1-s}, \\
G & \equiv G_{\Phi, s} \equiv\left(\Phi^{-1}(\varrho)\right)^{s} g\left(\Phi^{-1}(\varrho)\right)^{1-s} .
\end{aligned}
$$

Since

$$
O_{\Phi, s}(\alpha f+(1-\alpha) g)=\operatorname{Tr}(\Phi(|\alpha F+(1-\alpha) G|)),
$$

using the fact that by our assumption $\Phi$ is a Young function together with Lemmata 2.1 and 2.2 we have

$$
\begin{aligned}
\operatorname{Tr}(\Phi(|\alpha F+(1-\alpha) G|)) & \leq \alpha \operatorname{Tr}\left(\Phi\left(u^{*}|F| u\right)\right)+(1-\alpha) \operatorname{Tr}\left(\Phi\left(v^{*}|G| v\right)\right) \\
& \leq \alpha \operatorname{Tr}\left(u^{*} \Phi(|F|) u\right)+(1-\alpha) \operatorname{Tr}\left(v^{*} \Phi(|G|) v\right) \\
& \leq \alpha \operatorname{Tr}(\Phi(|F|))+(1-\alpha) \operatorname{Tr}(\Phi(|G|)) .
\end{aligned}
$$

Since $\alpha \operatorname{Tr}(\Phi(|F|))+(1-\alpha) \operatorname{Tr}(\Phi(|G|))=\alpha O_{\Phi, s}(f)+(1-\alpha) O_{\Phi, s}(g)$, this implies the absolute convexity of $K_{\Phi, s}$.

(ii) We need to show that the sum of two operators in $L_{\Phi, s}(\mathcal{A}, \omega)$ also belongs to $L_{\Phi, s}(\mathcal{A}, \omega)$. For $i=1,2$, let $h_{i} \in L_{\Phi, s}(\mathcal{A}, \omega)$; that is, by definition, there exist $\beta_{i} \in(0,1]$ such that $\beta_{i} h_{i} \in K_{\Phi, s}$. Let $\beta=\min \left(\beta_{1}, \beta_{2}\right)$. Then $\beta>0$ and setting $H_{i}=\left(\Phi^{-1}(\varrho)\right)^{s} h_{i}\left(\Phi^{-1}(\varrho)\right)^{1-s}$ we have

$$
\begin{aligned}
O_{\Phi, s}\left(\frac{\beta}{2}\left(h_{1}+h_{2}\right)\right) & =\operatorname{Tr}\left[\Phi\left(\left|\frac{\beta}{2}\left(H_{1}+H_{2}\right)\right|\right)\right] \\
& \leq \frac{1}{2}\left(\operatorname{Tr}\left(\Phi\left(\left|\beta H_{1}\right|\right)\right)+\operatorname{Tr}\left(\Phi\left(\left|\beta H_{2}\right|\right)\right)\right) \\
& \leq \frac{1}{2}\left[O_{\Phi, s}\left(\beta_{1} h_{1}\right)+O_{\Phi, s}\left(\beta_{2} h_{2}\right)\right] \leq 1
\end{aligned}
$$


(where we have used monotonicity and convexity properties of $\Phi$ ). Hence, $h_{1}+h_{2} \in L_{\Phi, s}(\mathcal{A}, \omega)$. The fact that a scalar multiple of an operator in $L_{\Phi, s}(\mathcal{A}, \omega)$ belongs to $L_{\Phi, s}(\mathcal{A}, \omega)$ follows from the remark after the definition of this set.

We furnish $L_{\Phi, s}(\mathcal{A}, \omega)$ with the Luxemburg norm

$$
\|f\|_{\Phi, s}:=\inf \left\{\lambda>0: \frac{1}{\lambda} f \in K_{\Phi, s}\right\} .
$$

One can show the following facts.

Proposition 3.2.

(i) $\left(L_{\Phi, s}(\mathcal{A}, \omega),\|\cdot\|_{\Phi, s}\right)$ is a normed linear space.

(ii) If $\|f\|_{\Phi, s} \leq 1$, then $O_{\Phi, s}(f) \leq\|f\|_{\Phi, s}$, with equality if the norm equals 1 .

Given $s \in[0,1]$ we introduce the scalar product

$$
\langle f, g\rangle_{s}:=\operatorname{Tr}\left(\varrho^{1-s} f^{*} \varrho^{s} g\right) .
$$

It is useful to note the following representation of the scalar product.

LEMMA 3.3.

$$
\langle f, g\rangle_{s}=\operatorname{Tr}\left(M^{1-s} F_{\Phi, s}^{*} M^{s} G_{\Psi, s}\right)
$$

with a bounded operator

$$
1 / 2 \leq M \equiv \varrho\left(\Phi^{-1}(\varrho)\right)^{-1}\left(\Psi^{-1}(\varrho)\right)^{-1} \leq 1 .
$$

Proof. Since we have $F_{\Phi, s}^{*} \equiv\left(\Phi^{-1}(\varrho)\right)^{1-s} f^{*}\left(\Phi^{-1}(\varrho)\right)^{s}$ and $G_{\Psi, s} \equiv$ $\left(\Psi^{-1}(\varrho)\right)^{s} g\left(\Psi^{-1}(\varrho)\right)^{1-s}$, using the definition of the scalar product, we obtain

$$
\begin{aligned}
\langle f, g\rangle_{s}= & \operatorname{Tr}\left(\varrho^{1-s} f^{*} \varrho^{s} g\right) \\
= & \operatorname{Tr}\left(\left(\Phi^{-1}(\varrho)\right)^{1-s} f^{*}\left(\Phi^{-1}(\varrho)\right)^{s}\left\{\left(\Phi^{-1}(\varrho)\right)^{-s} \varrho^{s}\left(\Psi^{-1}(\varrho)\right)^{-s}\right\}\right. \\
& \left.\left.\cdot\left(\Psi^{-1}(\varrho)\right)^{s} g\left(\Psi^{-1}(\varrho)\right)^{1-s}\left\{\left(\Psi^{-1}(\varrho)\right)^{-(1-s)}\right) \varrho^{1-s}\left(\Phi^{-1}(\varrho)\right)^{-(1-s)}\right\}\right) \\
= & \operatorname{Tr}\left(F_{\Phi, s}^{*} M^{s} G_{\Psi, s} M^{1-s}\right)=\operatorname{Tr}\left(M^{1-s} F_{\Phi, s}^{*} M^{s} G_{\Psi, s}\right) .
\end{aligned}
$$

The lower and upper bounds of $M$ follow from (1).

We show the following relations between this scalar product and the functionals introduced above.

THEOREM 3.4.

(i) (Young inequality) For $f, g \in \mathcal{M}(\mathcal{A})$ and any density matrix $\varrho>0$,

$$
\left|\langle f, g\rangle_{s}\right| \leq O_{\Phi, s}(f)+O_{\Psi, s}(g) .
$$

(ii) (Hölder inequality)

$$
\left|\langle f, g\rangle_{s}\right| \leq 2\|f\|_{\Phi, s}\|g\|_{\Psi, s}
$$

for any $f \in L_{\Phi, s}(\mathcal{A}, \omega)$ and $g \in L_{\Psi, s}(\mathcal{A}, \omega)$. 
Proof. (i) For simplicity of notation, setting $F \equiv F_{\Phi, s}$ and $G \equiv G_{\Psi, s}$, from Lemma 3.3 we have

$$
\langle f, g\rangle_{s}=\operatorname{Tr}\left(M^{1-s} F^{*} M^{s} G\right) .
$$

Applying the absolute value and using the Young inequality for traces (Lemma 2.2(3)), we get

$$
\begin{aligned}
\left|\langle f, g\rangle_{s}\right| & =\left|\operatorname{Tr}\left(M^{1-s} F^{*} M^{s} G\right)\right| \leq \operatorname{Tr}\left(\left|M^{1-s} F^{*} M^{s} G\right|\right) \\
& \leq \operatorname{Tr}\left(\Phi\left(\left|M^{1-s} F^{*}\right|\right)\right)+\operatorname{Tr}\left(\Psi\left(\left|M^{s} G\right|\right)\right) .
\end{aligned}
$$

Next we use Lemma 2.1(4) to represent each term from the right hand side of (3) as the integral of a monotone function of the corresponding rearrangement. At this stage we utilize properties (1)-(3) of Lemma 2.1 together with the upper bound $M \leq 1$ of Lemma 2.2 to get

$$
\begin{aligned}
\left|M^{1-s} F^{*}\right|^{\sim} & \leq\left\|M^{1-s}\right\| \cdot|F|^{\sim} \leq|F|^{\sim}, \\
\left|M^{s} G\right|^{\sim} & \leq\left\|M^{s}\right\| \cdot|G|^{\sim} \leq|G|^{\sim} .
\end{aligned}
$$

This implies the bound

$$
\begin{aligned}
\left|\langle f, g\rangle_{s}\right| & \leq\left\|M^{1-s}\right\| \operatorname{Tr}\left(\Phi\left(\left|F^{*}\right|\right)\right)+\left\|M^{s}\right\| \operatorname{Tr}(\Psi(|G|)) \\
& \leq \operatorname{Tr}\left(\Phi\left(\left|F^{*}\right|\right)\right)+\operatorname{Tr}(\Psi(|G|))=\operatorname{Tr}(\Phi(|F|))+\operatorname{Tr}(\Psi(|G|)) .
\end{aligned}
$$

Now it is sufficient to recall our definition of $F$ and $G$ to arrive at the desired Young inequality

$$
\left|\langle f, g\rangle_{s}\right| \leq O_{\Phi, s}(f)+O_{\Psi, s}(g) .
$$

(ii) For $f \in L_{\Phi, s}(\mathcal{A}, \omega)$ and $g \in L_{\Psi_{s}}(\mathcal{A}, \omega)$ with $\|f\|_{\Phi, s},\|g\|_{\Psi_{s}} \neq 0$, we have $O_{\Phi, s}\left(f /\|f\|_{\Phi, s}\right)=1=O_{\Psi, s}\left(g /\|g\|_{\Psi_{s}}\right)$. Therefore by the Young inequality,

$$
\left|\left\langle\frac{f}{\|f\|_{\Phi, s}}, \frac{g}{\|g\|_{\Psi_{s}}}\right\rangle_{s}\right| \leq O_{\Phi, s}\left(\frac{f}{\|f\|_{\Phi, s}}\right)+O_{\Psi, s}\left(\frac{g}{\|g\|_{\Psi_{s}}}\right)=2 .
$$

From this the desired inequality follows.

In the rest of this section we introduce other natural norms on $L_{\Phi, s}(\mathcal{A}, \omega)$ and discuss their relations. We have already introduced the definition of the Luxemburg norm which can be represented in the following equivalent form:

$$
\|f\|_{\Phi, s}=\inf \left\{\lambda>0: O_{\Phi, s}(f / \lambda) \leq 1\right\}
$$

We define the Orlicz norm as follows:

$$
\|f\|_{\Phi, s}^{\circ}:=\sup \left\{\left|\langle f, g\rangle_{s}\right|:\|g\|_{\Psi, s} \leq 1\right\},
$$

where because of Proposition 3.2(ii) the condition $\|g\|_{\Psi, s} \leq 1$ can be replaced by $O_{\Psi, s}(g) \leq 1$. We also introduce the following prime norm:

$$
\|f\|_{\Phi, s}^{\prime}:=\inf _{k>0}\left\{\frac{1}{k}\left(1+O_{\Phi, s}(k f)\right)\right\} .
$$


We remark first that with these definitions one gets

$$
\begin{aligned}
\left|\langle f, g\rangle_{s}\right| & =\left|\left\langle f, g /\|g\|_{\Psi, s}\right\rangle_{s}\right|\|g\|_{\Psi, s} \\
& \leq \sup _{\|h\|_{\Psi, s} \leq 1}\left|\langle f, h\rangle_{s}\right|\|f\|_{\Phi, s}^{\circ}\|g\|_{\Psi, s}=\|f\|_{\Phi, s}^{\circ}\|g\|_{\Psi, s} .
\end{aligned}
$$

That is, we get another representation of the Hölder inequality:

Proposition 3.5.

$$
\left|\langle f, g\rangle_{s}\right| \leq\|f\|_{\Phi, s}^{\circ}\|g\|_{\Psi, s}
$$

Next recall that two norms, $\|\cdot\|_{1}$ and $\|\cdot\|_{2}$, on a normed linear space $X$ are called equivalent if there exist two positive real constants $C$ and $D$ such that $C\|\mathbf{x}\|_{1} \leq\|\mathbf{x}\|_{2} \leq D\|\mathbf{x}\|_{1}$ for all $\mathbf{x} \in X$. We show the following result proving the equivalence of the norms introduced above.

Theorem 3.6. For any $f \in L_{\Phi, s}(\mathcal{A}, \omega)$ one has

$$
\frac{1}{2}\|f\|_{\Phi, s} \leq\|f\|_{\Phi, s}^{\circ} \leq\|f\|_{\Phi, s}^{\prime} \leq 2\|f\|_{\Phi, s} .
$$

Proof of the first inequality of (9). First of all we recall that by the Young inequality for a trace ([12, Proposition 2.2]), for any measurable $G$ there is a $0 \leq b \in \mathcal{M}(\mathcal{A})$ satisfying $\operatorname{Tr}(\Psi(b)) \leq 1$ such that

$$
\operatorname{Tr}(|G| b)=\operatorname{Tr}(\Phi(|G|))+\operatorname{Tr}(\Psi(b)) .
$$

Thus for any $0<f \in L_{\Phi, s}(\mathcal{A}, \omega)$ with $F \equiv F_{\Phi, s}$ we have

$$
\begin{aligned}
O_{\Phi, s}(f) & =\operatorname{Tr}(\Phi(|F|)) \leq \operatorname{Tr}(\Phi(|F|))+\operatorname{Tr}(\Psi(b)) \\
& =\operatorname{Tr}(\Phi(|F|))+\operatorname{Tr}(\Psi(b))=\operatorname{Tr}\left(\left|F^{*}\right| b\right)
\end{aligned}
$$

with a suitable $0 \leq b \in \mathcal{M}(\mathcal{A})$ satisfying $\operatorname{Tr}(\Psi(b)) \leq 1$. The polar decomposition $F^{*}=W\left|F^{*}\right|$ implies $W^{*} F^{*}=\left|F^{*}\right|$, where $W$ is the partial isometry for $F^{*}$. Hence the right hand side of (10) can be written as

$$
\operatorname{Tr}\left(\left|F^{*}\right| b\right)=\operatorname{Tr}\left(W^{*} F^{*} b\right)=\operatorname{Tr}\left(F^{*} b W^{*}\right)
$$

by cyclicity of trace. Inserting the explicit expression for $F \equiv F_{\Phi, s}$, after simple manipulations under the trace we get

$$
\begin{aligned}
\operatorname{Tr}\left(\left|F^{*}\right| b\right) & =\operatorname{Tr}\left(F^{*} b W^{*}\right) \\
& =\operatorname{Tr}\left(\left(\Phi^{-1}(\varrho)\right)^{1-s} f^{*}\left(\Phi^{-1}(\varrho)\right)^{s} b W^{*}\right) \\
& =\operatorname{Tr}\left(\left(\Phi^{-1}(\varrho)\right)^{1-s} \varrho^{-(1-s)} \varrho^{1-s} f^{*} \varrho^{s} \varrho^{-s}\left(\Phi^{-1}(\varrho)\right)^{s} b W^{*}\right) \\
& =\operatorname{Tr}\left(\left(\varrho^{1-s} f^{*} \varrho^{s}\right)\left(\varrho^{-s}\left(\Phi^{-1}(\varrho)\right)^{s} b W^{*} \varrho^{-(1-s)}\left(\Phi^{-1}(\varrho)\right)^{1-s}\right)\right)
\end{aligned}
$$

Inserting $\left(\Psi^{-1}(\varrho)\right)^{-s}\left(\Psi^{-1}(\varrho)\right)^{s}$ and $\left(\Psi^{-1}(\varrho)\right)^{-(1-s)}\left(\Psi^{-1}(\varrho)\right)^{1-s}$ on the left and on the right of the term in the second bracket under the trace on the 
right hand side of (12), respectively, we arrive at

$$
\begin{array}{r}
\operatorname{Tr}\left(\left|F^{*}\right| b\right)=\operatorname{Tr}\left(\left(\varrho^{1-s} f^{*} \varrho^{s}\right)\left(\Psi^{-1}(\varrho)\right)^{-s}\left(\left(\Psi^{-1}(\varrho)\right)^{s} \varrho^{-s}\left(\Phi^{-1}(\varrho)\right)^{s}\right) b W^{*}\right. \\
\left.\cdot\left(\varrho^{-(1-s)}\left(\Phi^{-1}(\varrho)\right)^{1-s}\left(\Psi^{-1}(\varrho)\right)^{1-s}\right)\left(\Psi^{-1}(\varrho)\right)^{-(1-s)}\right) \\
=\operatorname{Tr}\left(\left(\varrho^{1-s} f^{*} \varrho^{s}\right)\left(\Psi^{-1}(\varrho)\right)^{-s} M^{-s} b W^{*} M^{-(1-s)}\left(\Psi^{-1}(\varrho)\right)^{-(1-s)}\right) \\
=\operatorname{Tr}\left(\left(\varrho^{1-s} f^{*} \varrho^{s}\right)\left(\Psi^{-1}(\varrho)\right)^{-s} M^{-s} b W^{*} M^{-(1-s)}\left(\Psi^{-1}(\varrho)\right)^{-(1-s)}\right)
\end{array}
$$

where $M^{-1} \equiv \varrho^{-1} \Phi^{-1}(\varrho) \Psi^{-1}(\varrho)$. Setting

$$
\mathbf{b} \equiv \frac{1}{2}\left(\Psi^{-1}(\varrho)\right)^{-s} M^{-s} b W^{*} M^{-(1-s)}\left(\Psi^{-1}(\varrho)\right)^{-(1-s)},
$$

we can write (13) as

$$
\operatorname{Tr}\left(\left|F^{*}\right| b\right)=2\langle f, \mathbf{b}\rangle_{s} .
$$

Combining this with (10), we get

$$
O_{\Phi, s}(f) \leq 2\langle f, \mathbf{b}\rangle_{s} .
$$

To conclude the proof we need to show that $O_{\Psi, s}(\mathbf{b}) \leq 1$. To this end we note that

$$
\begin{aligned}
O_{\Psi, s}(\mathbf{b})= & \operatorname{Tr}\left(\Psi\left(\left|\left(\Psi^{-1}(\varrho)\right)^{s} \mathbf{b}\left(\Psi^{-1}(\varrho)\right)^{1-s}\right|\right)\right) \\
= & \operatorname{Tr}\left(\Psi \left(\mid\left(\Psi^{-1}(\varrho)\right)^{s}\right.\right. \\
& \left.\left.\quad \cdot\left(\Psi^{-1}(\varrho)\right)^{-s} \frac{1}{2} M^{-s} b W^{*} M^{-(1-s)}\left(\Psi^{-1}(\varrho)\right)^{-(1-s)}\left(\Psi^{-1}(\varrho)\right)^{1-s} \mid\right)\right) \\
\leq & \operatorname{Tr}\left(\Psi\left(\left|\frac{1}{2} M^{-s} b W^{*} M^{-(1-s)}\right|\right)\right) .
\end{aligned}
$$

Because by (1) we have $M^{-1} \leq 2$, using Lemma 2.1 we get the following inequality for rearrangements:

$$
\left|\frac{1}{2} M^{-s} b W^{*} M^{-(1-s)}\right|^{\sim} \leq \widetilde{b}
$$

This together with monotonicity of $\Psi$ on the positive real axis and our assumptions about $b$ implies that

$$
O_{\Psi, s}(\mathbf{b}) \leq \operatorname{Tr}\left(\Psi\left(\left|\frac{1}{2} M^{-s} b W^{*} M^{-(1-s)}\right|\right)\right) \leq \operatorname{Tr}(\Psi(b)) \leq 1 .
$$

From (14) and (15), we obtain

$$
O_{\Phi, s}(f) \leq 2\langle f, \mathbf{b}\rangle_{s} \leq 2\|f\|_{\Phi, s}^{\circ} .
$$

Applying this to a function with the Luxemburg norm equal to one and taking into account Proposition 3.2(ii), after simple algebraic manipulations we conclude with

$$
\frac{1}{2}\|f\|_{\Phi, s} \leq\|f\|_{\Phi, s}^{\circ} .
$$

Proof of the second inequality of (9). Let $g \in L_{\Psi, s}(\mathcal{A}, \omega)$. By definition of $\|f\|_{\Phi, s}^{\circ}$ (and Proposition 3.2(ii)), we have

$$
\|f\|_{\Phi, s}^{\circ}=\sup \left\{\left|\langle f, g\rangle_{s}\right|: O_{\Psi, s}(g) \leq 1\right\} .
$$


Hence for any $0<k<\infty$ using the Young inequality, one gets

$$
\begin{aligned}
\|f\|_{\Phi, s}^{\circ} & =\sup \left\{\left|\frac{1}{k}\langle k f, g\rangle_{s}\right|: O_{\Psi, s}(g) \leq 1\right\} \\
& \leq \sup \left\{\frac{1}{k}\left(O_{\Phi, s}(k f)+O_{\Psi, s}(g)\right): O_{\Psi, s}(g) \leq 1\right\} \\
& \leq \frac{1}{k}\left(O_{\Phi, s}(k f)+1\right) .
\end{aligned}
$$

Since $k \in(0, \infty)$ was arbitrary, taking the inf with respect to $k$ we arrive at

$$
\|f\|_{\Phi, s}^{\circ} \leq \inf _{k>0} \frac{1}{k}\left(O_{\Phi, s}(k f)+1\right) \equiv\|f\|_{\Phi, s}^{\prime} .
$$

(In fact one can show equality in this case.)

Proof of the third inequality of (9). Let $f \in \mathcal{M}(\mathcal{A})$ be such that $\|f\|_{\Phi, s}$ $\neq 0$. Using the definition of $\|\cdot\|_{\Phi, s}^{\prime}$, we have

$$
\begin{aligned}
\left\|\frac{f}{\|f\|_{\Phi, s}}\right\|_{\Phi, s}^{\prime} & =\inf _{k>0}\left\{\frac{1}{k}\left(1+O_{\Phi, s}\left(\frac{k f}{\|f\|_{\Phi, s}}\right)\right)\right\} \\
& \leq \frac{1}{\tilde{k}}\left(1+O_{\Phi, s}\left(\frac{\tilde{k} f}{\|f\|_{\Phi, s}}\right)\right)
\end{aligned}
$$

for any $\tilde{k}>0$. Choosing $\tilde{k}=1$ and noting that by Proposition 3.2(ii) one has $O_{\Phi, s}\left(f /\|f\|_{\Phi, s}\right)=1$, we arrive at

$$
\|f\|_{\Phi, s}^{\prime} \leq 2\|f\|_{\Phi, s} \text {. }
$$

4. Summary and outlook. We have introduced the Orlicz spaces associated to a given faithful normal state (or weight) on a semisimple von Neumann algebra. Our spaces generalise the well known noncommutative $L_{p}$ spaces. In a similar manner to that well established case, for every value of a continuous parameter $s \in[0,1]$ we can define a norm $\|\cdot\|_{\Phi, s}$ as well as its dual $\|\cdot\|_{\Psi, s}$ associated to the complementary Young function, which are related in a natural way to a scalar product $\langle\cdot, \cdot\rangle_{s}$. In certain applications it is useful to consider the following Bogolyubov-Kubo-Mori scalar product:

$$
\langle\langle f, g\rangle\rangle:=\int_{0}^{1}\langle f, g\rangle_{s} d s .
$$

It is then natural to consider the generalisation of the presented scheme in which for every $s$ one considers a pair of complementary Young functions $\left(\Phi_{s}, \Psi_{s}\right)$ depending possibly in a nontrivial way on $s$. Assuming that the dependence on $s$ in the family $\boldsymbol{\Phi}=\left(\Phi_{s}\right)_{s \in[0,1]}$ is sufficiently smooth, we can 
define a convex functional

$$
\boldsymbol{O}_{\boldsymbol{\Phi}}(f):=\int_{0}^{1} O_{\Phi_{s}, s}(f) d s
$$

and similarly for the complementary family $\boldsymbol{\Psi}=\left(\Psi_{s}\right)_{s \in[0,1]}$. With such functionals one can recover all elements of the theory discussed in the previous section, including in particular the Young inequality

$$
\langle\langle f, g\rangle\rangle \leq \boldsymbol{O}_{\boldsymbol{\Phi}}(f)+\boldsymbol{O}_{\boldsymbol{\Psi}}(f) .
$$

Some applications of our theory will be described elsewhere.

\section{References}

[1] C. Akemann, J. Anderson and G. Pedersen, Triangle inequalities in operator algebras, Linear Multilinear Algebra 11 (1982), 167-178.

[2] H. Araki and T. Masuda, Positive cones and $L_{p}$-spaces of von Neumann algebras, Publ. R.I.M.S. Kyoto Univ. 18 (1982), 339-411.

[3] R. Bhatia, Matrix Analysis, Springer, New York, 1997.

[4] L. G. Brown and H. Kosaki, Jensen's inequality in semi-finite von Neumann algebras, J. Operator Theory 23 (1990), 3-19.

[5] J. Dixmier, Formes linéaires sur un anneau d'opérateurs, Bull. Soc. Math. France 81 (1953), 9-39.

[6] T. Fack and H. Kosaki, Generalised s-numbers of $\tau$-measurable operators, Pacific J. Math. 123 (1986), 269-300.

[7] U. Haagerup, $L_{p}$-spaces associated with an arbitrary von Neumann algebra, in: Algèbres d'opérateurs et leurs applications en physique mathématique (Marseille, 1977), Colloq. Internat. CNRS 274, Éditions du CNRS, Paris, 1979, 175-184.

[8] M. Hilsum, Les espaces $L_{p}$ d'une algèbre de von Neumann définies par la dérivée spatiale, J. Funct. Anal. 40 (1981), 151-169.

[9] H. Kosaki, Application of the complex interpolation method to a von Neumann algebra (Non-commutative $L_{p}$-spaces), ibid. 56 (1984), 29-78.

[10] A. Kufner and S. Fučík, Function Spaces, Academia, Prague, 1977.

[11] R. A. Kunze, $L_{p}$ Fourier transforms on locally compact unimodular groups, Trans. Amer. Math. Soc. 89 (1958), 519-540.

[12] W. Kunze, Noncommutative Orlicz spaces and generalized Arens algebras, Math. Nachr. 147 (1990), 123-138.

[13] M. Muratov, Noncommutative Orlicz spaces, Dokl. Akad. Nauk UzSSR 1978, no. 6, 11-13 (in Russian).

[14] -, The Luxemburg norm in an Orlicz space of measurable operators, ibid. 1979, no. 1, 5-6 (in Russian).

[15] E. Nelson, Notes on non-commutative integration, J. Funct. Anal. 15 (1974), 103116.

[16] M. Rao and Z. Ren, Theory of Orlicz Spaces, Dekker, New York, 1991.

[17] S. Sakai, $C^{*}$-Algebras and $W^{*}$-Algebras, Springer, 1971.

[18] I. E. Segal, A non-commutative extension of abstract integration, Ann. of Math. 57 (1953), 401-457. 
[19] M. Terp, $L_{p}$-spaces associated with von Neumann algebras, Københavns Universitet, Mathematisk Institut, Rapport No. 3 (1981).

[20] - Interpolation spaces between a von Neumann algebra and its dual, J. Operator Theory 8 (1982), 327-360.

[21] N. V. Trunov, On a noncommutative analogue of the space $L_{p}$, Izv. VUZ Mat. 1979, no. 11, 69-77 (in Russian); English transl.: Soviet Math. 23 (1979), no. 11, 71-79.

[22] F. J. Yeadon, Non-commutative $L_{p}$-spaces, Math. Proc. Cambridge Philos. Soc. 77 (1975), 91-102.

Department of Mathematics

Imperial College

180 Queen's Gate

London SW7 2AZ, UK

E-mail: b.zegarlinski@imperial.ac.uk

Received July 3, 2003

Revised version March 3, 2004 